\title{
In-Field Calibration of a Multi-Mode Antenna for DoA Estimation
}

\author{
Robert Pöhlmann*, Emanuel Staudinger*, Siwei Zhang*, Stefano Caizzone*, Armin Dammann*, Peter A. Hoeher ${ }^{\dagger}$ \\ ${ }^{*}$ Institute of Communications and Navigation, German Aerospace Center (DLR), Oberpfaffenhofen Germany, \\ \{robert.poehlmann, emanuel.staudinger, siwei.zhang, stefano.caizzone, armin.dammann\}@dlr.de \\ ${ }^{\dagger}$ Faculty of Engineering, University of Kiel, Kiel, Germany, ph@tf.uni-kiel.de
}

\begin{abstract}
State-of-the-art radio localization methods often rely on estimating the signal direction-of-arrival (DoA) or directionof-departure (DoD). This requires careful calibration of the antenna, including surrounding structure, in a measurement chamber. Alternatively, auto-calibration algorithms can be used for certain types of phased arrays. However, they cannot correct all model errors and cannot be applied to other types of multiport antennas like multi-mode antennas (MMAs) or collocated antennas. In this paper, we present a maximum a posteriori (MAP) in-field calibration algorithm based on wavefield modeling and manifold separation for arbitrary multiport antennas. The algorithm is evaluated using measurements of a four-port MMA mounted on a rover. A significant performance gain for DoA estimation using in-field calibration is achieved compared to calibration in a chamber.
\end{abstract}

Index Terms-multi-mode antenna, in-field calibration, DoA estimation, antenna calibration, maximum a posteriori

\section{INTRODUCTION}

Radio-based localization in global navigation satellite system (GNSS) denied areas or in addition to GNSS is a very active research topic. Applications range from cellular networks [1] over autonomous driving [2] to extraterrestrial exploration [3], [4]. Based on the observation type, localization methods can be classified into three categories. Distance-based methods use e.g. the signal time-of-flight (ToF) in synchronized or the round-trip time (RTT) in unsynchronized networks to determine the distance between two nodes. Based on distance estimates, the position is determined by trilateration. Anglebased methods use the signal DoA or DoD and estimate the position by triangulation. The third type are hybrid methods, combining both observation types. Angle-based methods usually rely on phased arrays [5] to estimate DoA or DoD. More recently, DoA estimation with a single MMA has been proposed [6].The estimation performance crucially depends on exact knowledge of the antenna response, independent of used antenna type. To obtain the antenna response, antennas are usually measured in anechoic chambers. Especially when the antenna is mounted on a larger surrounding structure, e.g. a rover or a flying platform, this can become costly and impractical.

Instead, antenna auto-calibration or self-calibration can be performed. The unknown antenna parameters to be determined are considered deterministic [7] or stochastic with a known prior distribution [8], [9]. The idea is to estimate both antenna and wavefield parameters (i.e. the DoAs) at the same time. The challenge for both deterministic and stochastic approaches is the identifiability issue [10]. In general, antenna or array parameters cannot be estimated together with the wavefield. To solve this, the methods have to make strong assumptions about the underlying physics. When all antenna ports have the same gain pattern, their phase patterns can be determined [11]. Assuming a uniform linear array (ULA), gain and phase patterns can be estimated [10]. Real-world multiport antennas often differ from these assumptions, which leads to a model mismatch and degraded performance. Moreover, the methods are limited to phased arrays. A first approach generalizing infield calibration is presented in [12]. However, an exact DoA reference is required and the work is based on simulation only.

In this paper, we present a MAP algorithm for in-field calibration of arbitrary multiport antennas, including phased arrays, MMAs and collocated antennas [13]. The algorithm is initialized with an a-priori known antenna response, which is then improved based on measurements and a noisy DoA reference. The model errors need not be linear. To illustrate its practical applicability, we present measurement results for a four-port MMA mounted on a rover. DoA estimation is performed with the antenna response determined by electromagnetic field (EMF) simulation, in a near-field measurement chamber and with the proposed in-field calibration algorithm, which is applied to measurement data obtained in an outdoor environment. By in-field calibration, the performance is improved considerably.

\section{SignAL MODEL}

A very generic description of a multi-port antenna, including an MMA, is in terms of their gain pattern $g_{m}(\phi)$ and phase pattern $\Phi_{m}(\phi)$ [14]. For port $m=1, \ldots, M$ and DoA $\phi$ the antenna response is defined as

$$
a_{m}(\phi)=\sqrt{g_{m}(\phi)} e^{\mathrm{j} \Phi_{m}(\phi)} .
$$

A total of $P$ superposed signals $\boldsymbol{s}(n)=\left[s_{1}(n), \ldots, s_{P}(n)\right]^{T}$ arrives from DoAs $\phi_{1}^{s}, \ldots, \phi_{P}^{s}$. We assume the antenna is connected to a multichannel receiver, which operates snapshotwise. During each snapshot $s$, the DoA $\phi^{s}$ is assumed to be constant, so we can write the sampled baseband signal, $\boldsymbol{r}^{s}(n)=\left[r_{1}(n), \ldots, r_{M}(n)\right]^{T}$, as

$$
\boldsymbol{r}^{s}(n)=\boldsymbol{A}\left(\phi^{s}\right) \boldsymbol{s}(n)+\boldsymbol{w}_{\boldsymbol{r}}(n),
$$


with sample index $n=1, \ldots, N$ and the antenna response matrix

$$
\boldsymbol{A}\left(\phi^{s}\right)=\left[\begin{array}{lll}
\boldsymbol{a}\left(\phi_{1}^{s}\right) & \ldots & \boldsymbol{a}\left(\phi_{P}^{s}\right)
\end{array}\right],
$$

which consists of antenna response vectors

$$
\boldsymbol{a}\left(\phi^{s}\right)=\left[\begin{array}{lll}
a_{1}\left(\phi^{s}\right) & \ldots & a_{M}\left(\phi^{s}\right)
\end{array}\right]^{T} .
$$

We adopt the narrowband assumption, i.e. the signal bandwidth is small relative to the carrier frequency [14], [15], and the system is assumed to be internally noise limited, such that $\boldsymbol{w}_{\boldsymbol{r}}(n) \sim \mathcal{C N}\left(0, \sigma_{\boldsymbol{r}}^{2} \mathbb{I}_{M}\right)$ is independent and identically distributed (i.i.d.) white circular symmetric Gaussian noise.

By wavefield modeling and manifold separation [16], [17] we decompose the antenna response vector,

$$
\boldsymbol{a}(\phi)=\boldsymbol{G} \boldsymbol{b}(\phi),
$$

into a product of the sampling matrix $G \in \mathbb{C}^{M \times U}$, which does not depend on the wavefield or DoA, and the basis vector $\boldsymbol{b}(\phi) \in \mathbb{C}^{U}$, which does not depend on the actual antenna. In [6] manifold separation has been applied to an MMA. This decomposition requires the antenna response to be square integrable and the basis functions of order $U$ to be orthonormal on the manifold $\phi \in[-\pi, \pi)$. The Fourier functions

$$
\boldsymbol{b}(\phi)=\frac{1}{\sqrt{2 \pi}} e^{\mathrm{j} \phi u_{\phi}}, u_{\phi}=\left\lfloor-\frac{U-1}{2}\right\rfloor, \ldots, 0, \ldots,\left\lfloor\frac{U-1}{2}\right\rfloor
$$

constitute a suitable basis. The necessary order $U$ is roughly linked to the electrical size of the antenna [16]. It can also be determined based on the measurement noise floor [18]. While manifold separation can be extended to 3D [16], [17], [6], where azimuth and elevation are to be estimated. In this paper, we focus on azimuth only.

The true sampling matrix $\boldsymbol{G}$ of a real antenna is in general unknown. A data driven approach is to measure the antenna in an anechoic chamber, yielding $Q$ spatial samples of the antenna response, $e_{q}=\left[e_{q, 1}, \ldots, e_{q, M}\right]^{T}$ for DoAs $\phi_{q}$ and $q=1, \ldots, Q$. In matrix form we have $\boldsymbol{E}^{\text {meas }}=\left[\boldsymbol{e}_{1}, \ldots, \boldsymbol{e}_{Q}\right]^{T}$. An approximate sampling matrix based on measurement data, $G^{\text {meas }}$, can then be calculated by least squares,

$$
\boldsymbol{G}^{\text {meas }}=\boldsymbol{E}^{\text {meas }} \boldsymbol{B}^{H}\left(\boldsymbol{B} \boldsymbol{B}^{H}\right)^{-1},
$$

with $\boldsymbol{B}=\left[\boldsymbol{b}\left(\phi_{1}\right), \ldots, \boldsymbol{b}\left(\phi_{Q}\right)\right]$. This approach is valid as long as the sampling grid is dense enough, such that the spatial Nyquist theorem is fulfilled. In the same fashion, a sampling matrix based on EMF simulation data $\boldsymbol{E}^{\mathrm{sim}}$,

$$
\boldsymbol{G}^{\operatorname{sim}}=\boldsymbol{E}^{\operatorname{sim}} \boldsymbol{B}^{H}\left(\boldsymbol{B} \boldsymbol{B}^{H}\right)^{-1},
$$

can be obtained.

However, EMF simulation does not account for manufacturing imperfections. A near-field measurement chamber is very limited in space, such that in this case it was only possible to measure the antenna without the surrounding assembly, see also Figure 1. For precise DoA estimation, very accurate knowledge of the antenna response is vital, as any model mismatch will degrade estimation performance. For these reasons, we propose to calibrate the antenna in the field, in order to capture the true antenna response of the MMA mounted on the assembly.

\section{IN-FIELD ANTENNA CALIBRATION}

Assuming $P=1$, the concentrated negative log-likelihood function [15] for snapshot $s$ is given by

$$
\tilde{L}\left(\hat{\boldsymbol{R}}^{s} \mid \phi, \boldsymbol{G}\right)=\frac{1}{2 \sigma_{\boldsymbol{r}}^{2}} \operatorname{Re}\left\{\operatorname{tr}\left\{\left(\mathbb{I}_{M}-\boldsymbol{a}(\phi) \boldsymbol{a}^{\dagger}(\phi)\right) \hat{\boldsymbol{R}}^{s}\right\}\right\},
$$

where $\dagger$ is the Moore-Penrose pseudoinverse and

$$
\hat{\boldsymbol{R}}^{s}=\sum_{n=1}^{N} \boldsymbol{r}^{s}(n)\left(\boldsymbol{r}^{s}(n)\right)^{H} .
$$

The maximum likelihood (ML) DoA estimator is then given by

$$
\hat{\phi}_{\mathrm{ML}}^{s}=\arg \min _{\phi} \tilde{L}\left(\hat{\boldsymbol{R}}^{s} \mid \phi, \boldsymbol{G}\right),
$$

and requires knowledge of the antenna response $\boldsymbol{a}(\phi)$, i.e. the sampling matrix $G$, see (5). In practice, the true sampling matrix is unknown, and errors in a measured or simulated sampling matrix result in a model mismatch, which impairs DoA estimation performance.

To cope with that, we adopt an approach from [9], where a MAP algorithm is proposed to estimate model errors of an array steering vector together with the DoA. We extend the approach, and propose a MAP algorithm together with wavefield modeling and manifold separation, making it suitable for any kind of real-world multiport antenna, e.g. phased array [5], MMA [6] or collocated antennas [13]. For the in-field calibration, we assume a DoA reference

$$
\phi_{\text {ref }}^{s}=\phi^{s}+w_{\phi_{\text {ref }}}
$$

is available, which is the true DoA $\phi$ with additive errors following a von Mises distribution $w_{\phi_{\text {ref }}} \sim \mathcal{M}\left(0, \kappa_{\phi_{\text {ref }}}\right)$ with concentration $\kappa_{\phi_{\text {ref }}}$. The concentration $\kappa_{\phi_{\text {ref }}}$ is expected to be high, so the distribution is approximated by a normal distribution $w_{\phi_{\text {ref }}} \sim \mathcal{N}\left(0, \sigma_{\phi_{\text {ref }}}^{2}=1 / \kappa_{\text {ref }}\right)$. Starting point for the algorithm is an a-priori known sampling matrix

$$
\boldsymbol{G}^{0}=\boldsymbol{G}+\boldsymbol{W}_{\boldsymbol{G}^{0}},
$$

which is the true sampling matrix $G$ subject to Gaussian model errors $\boldsymbol{W}_{\boldsymbol{G}^{0}}=\left[\boldsymbol{w}_{\boldsymbol{g}^{0}}^{1}, \ldots, \boldsymbol{w}_{\boldsymbol{g}^{0}}^{U}\right]$ with $\boldsymbol{w}_{\boldsymbol{g}^{0}}^{u} \sim \mathcal{C N}\left(0, \sigma_{\boldsymbol{G}^{0}}^{2} \mathbb{I}_{M}\right)$. In our case, we initialize the algorithm with the sampling matrix from the near-field measurements, i.e. $G^{0}=G^{\text {meas }}$. The MAP algorithm then jointly estimates the sampling matrix $\hat{\boldsymbol{G}}_{\mathrm{MAP}}^{S}$ together with the DoAs for all snapshots $\hat{\boldsymbol{\phi}}_{\mathrm{MAP}}^{S}=$ $\left[\hat{\phi}_{\mathrm{MAP}}^{1}, \ldots, \hat{\phi}_{\mathrm{MAP}}^{S}\right]^{T}$ and is given by

$$
\begin{aligned}
& \left\{\hat{\boldsymbol{G}}_{\mathrm{MAP}}^{S}, \hat{\boldsymbol{\phi}}_{\mathrm{MAP}}^{S}\right\}=\arg \min _{\boldsymbol{G}, \phi^{S}} \sum_{s=1}^{S} \tilde{L}\left(\hat{\boldsymbol{R}}^{s} \mid \phi^{s}, \boldsymbol{G}\right) \\
& \quad+\sum_{s=1}^{S} \frac{1}{2 \sigma_{\phi_{\mathrm{ref}}}^{2}}\left|\phi^{s}-\phi_{\mathrm{ref}}^{s}\right|^{2}+\frac{1}{2 \sigma_{\boldsymbol{G}^{0}}^{2}}\left\|\boldsymbol{G}-\boldsymbol{G}^{0}\right\|_{\mathrm{F}}^{2}
\end{aligned}
$$




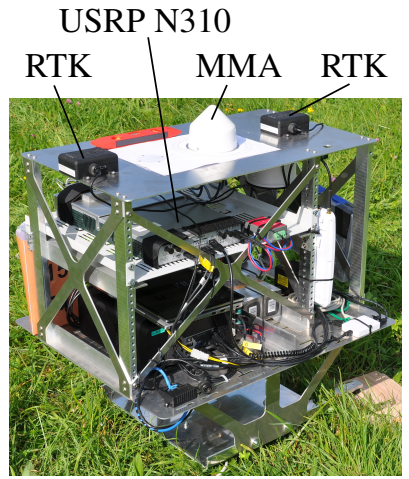

(a) Assembly on turntable.

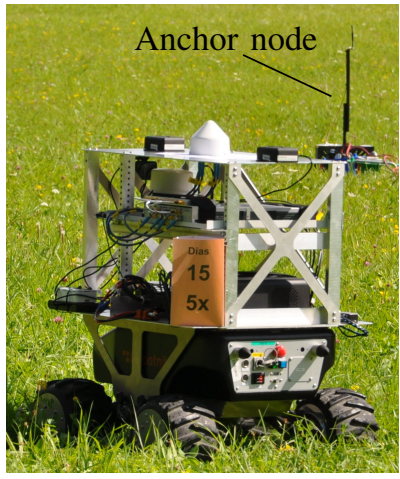

(b) Assembly on rover, anchor node in background.
Fig. 1. Assembly with installed MMA, multichannel SDR USRP N310, hos computer and two antenna RTK receiver for position and attitude reference.

with the Frobenius norm $\|.\|_{F}$. Solving (14) is a very challenging optimization problem. It is highly nonlinear and the total number of unknowns is $M U+S$. In order to solve it and avoid getting stuck in a local minimum, we adopt the following sequential procedure. We first solve for $\left\{\hat{\boldsymbol{G}}_{\mathrm{MAP}}^{1}, \hat{\boldsymbol{\phi}}_{\mathrm{MAP}}^{1}\right\}$. The result is then used to initialize the solver to obtain $\left\{\hat{G}_{\mathrm{MAP}}^{2}, \hat{\phi}_{\mathrm{MAP}}^{2}\right\}$, and so on, until the final solution $\left\{\hat{\boldsymbol{G}}_{\mathrm{MAP}}^{S}, \hat{\boldsymbol{\phi}}_{\mathrm{MAP}}^{S}\right\}$ is obtained. For each step, (14) is solved numerically using the Broyden-Fletcher-Goldfarb-Shanno (BFGS) algorithm [19].

\section{MEASUREMENT SETUP}

The employed dielectric resonator antenna was developed at DLR and features four modes, which are excited independently. Details can be found in [20]. To perform manifold separation in (5), $U=13$ is chosen as order of the basis functions (6).

The physical layer is defined by the DLR Swarm Communication and Navigation system [21]. The system uses $1.68 \mathrm{GHz}$ carrier frequency, $31.25 \mathrm{MHz}$ bandwidth and orthogonal frequency-division multiplexing (OFDM) with symbol length 1024 samples. Channel access is realized with a selforganizing time-division multiple access (TDMA) scheme with $100 \mathrm{~ms}$ round-trip schedule. The transmit power is set to $-15 \mathrm{dBm}$. More details about the system can be found in [21], [22].

The system is implemented as SDR using GNU Radio and Ettus Research B200mini devices for the single channel nodes. For MMAs we know that DoA information is contained in the magnitude and phase of the received signal, see (1). This means we need a phase-coherent multichannel receiver, where the phase and amplitude imbalances between the channels are properly corrected. We employ the Universal Software Radio Peripheral (USRP) N310 from Ettus Research, where the local oscillator (LO) is provided by an external frequency synthesizer to to obtain four phase coherent channels [22].

The MMA together with the USRP N310, host computer, Wi-Fi transceiver, batteries and two GNSS real-time kinematic
(RTK) receivers are integrated in one assembly. The whole assembly can either be mounted on a turntable, see Figure 1a, or on a rover as in Figure 1b. The commercial multi-sensor RTK system [23] provides an orientation and position reference based on fused GNSS and inertial observations. The output, together with known anchor positions, is used to calculate the reference DoA $\phi_{\text {ref. }}$.

\section{Measurement Results}

Figure 2 shows three different power and phase patterns of the four ports of the MMA. The first one is obtained by an EMF simulation, where the antenna alone is simulated in free space. For the second set of patterns, the antenna is measured by an MVG Starlab near-field measurement system. The mounting structure and the radome of the manufactured antenna were not part of the simulation. Especially for the phase patterns, a notable difference is visible between simulation and measurement.

The third set of patterns is obtained by the proposed infield calibration algorithm. The MMA assembly is mounted on a turntable, see Figure 1a, which is placed in between three anchor nodes. A total of $S=375$ snapshots, 125 from each anchor, obtained during one full turn are used to run the in-field calibration algorithm presented in Section III. Each snapshot consists of two OFDM symbols, i.e. $N=2048$ samples. In this case, a total of 427 unknowns need to be determined to solve (14). The patterns obtained by in-field calibration are similar to the measured ones, but differences are visible in terms of both power and phase.

To evaluate the DoA estimation performance, the MMA assembly is mounted on a rover, see Figure $1 \mathrm{~b}$. For $10 \mathrm{~min}$ the rover drives in-between and around the three anchors with a velocity of $0.4 \sim 0.65 \mathrm{~m} / \mathrm{s}$. The track can be seen in Figure 3; the starting point for the rover is in the lower left corner. During this time, 5787 snapshots were received from A1, 5466 from A2, 5211 from A3, and recorded for post-processing. Based on this data, the signal DoA is estimated by the ML estimator (9) and (11) with the respective antenna response, i.e. $\boldsymbol{a}(\phi)=\boldsymbol{G}^{\mathrm{sim}} \boldsymbol{b}(\phi)$ for the simulated antenna response, $\boldsymbol{a}(\phi)=\boldsymbol{G}^{\text {meas }} \boldsymbol{b}(\phi)$ for the antenna response measured in the near-field chamber and $\boldsymbol{a}(\phi)=\hat{\boldsymbol{G}}_{\mathrm{MAP}}^{S} \boldsymbol{b}(\phi)$ for the antenna response obtained by the in-field calibration algorithm from Section III.

In Figure 4 , the DoA estimation error $\hat{\phi}_{\mathrm{ML}}-\phi_{\text {ref }}$ for the signals received from A3 is shown over time. Using the simulated antenna response, error peaks larger than $25^{\circ}$ are visible. With the measured antenna response, the error peaks are reduced to around $12^{\circ}$. Using the antenna response from in-field calibration, the error is reduced further; most of the peaks are below $8^{\circ}$. During the experiment, the rover was driving on bumpy grassland. This caused some shaking of the rover, which could explain the peaks in the estimation error.

The empirical cumulative distribution functions (CDFs) of the DoA estimation error with respect to anchors A1, A2 and A3 again using the three different antenna responses are plotted in Figure 5. Using the measured antenna response, $90 \%$ 

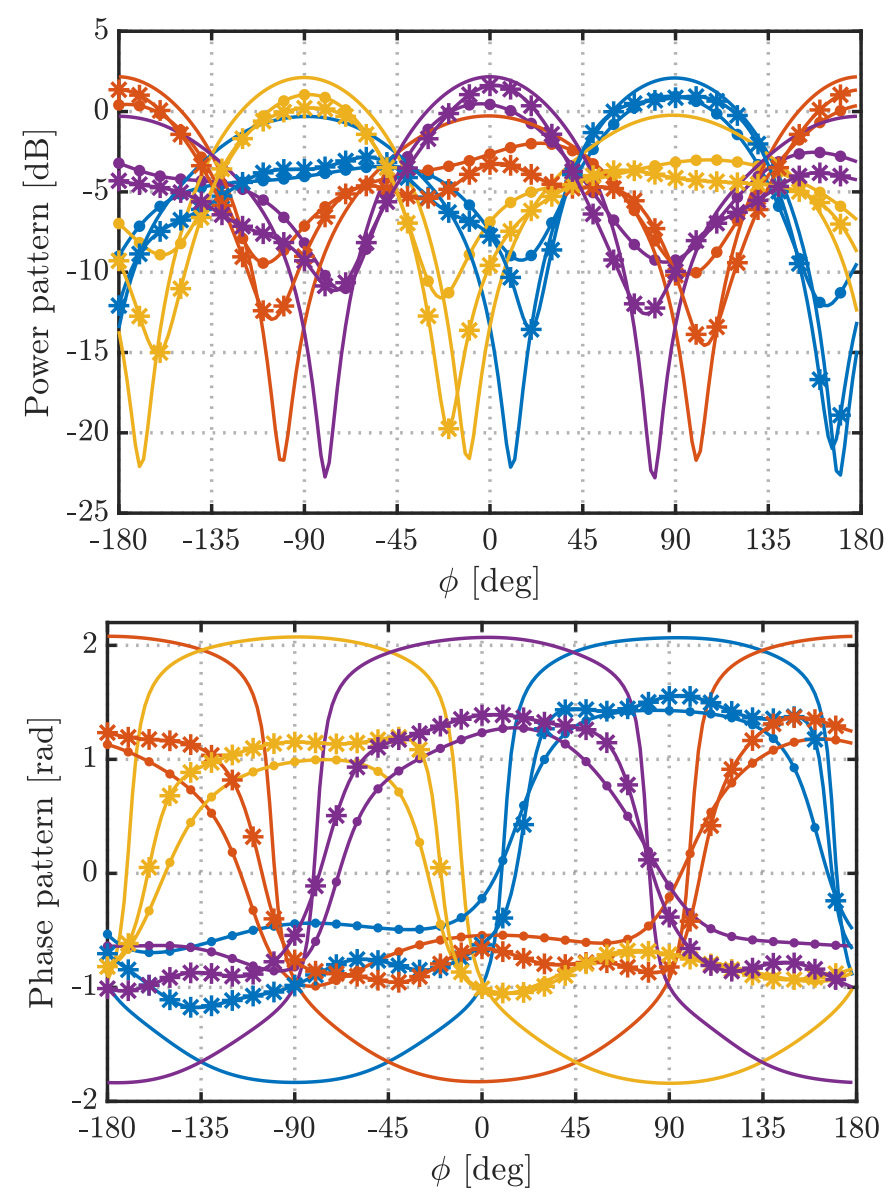

\begin{tabular}{|rr|r|}
\hline Sim. $1 \longrightarrow$ Meas. $1 \longrightarrow$ In-field 1 \\
Sim. $2 \longrightarrow$ Meas. $2 \longrightarrow$ In-field 2 \\
Sim. $3 \longrightarrow$ Meas. $3 \longrightarrow$ In-field 3 \\
Sim. $4 \longrightarrow$ Meas. $4 \longrightarrow$ In-field 4 \\
\hline
\end{tabular}

Fig. 2. Power pattern and phase pattern of ports 1-4 of the MMA obtained by EMF simulation (Sim.), in a near-field measurement chamber (Meas.) and by the in-field calibration algorithm from Section III (In-field).

of the measurement errors wrt. A1 are below $10.4^{\circ}$, compared to $7.9^{\circ}$ for the antenna response from in-field calibration. The signals from both A2 and A3 show lower DoA estimation errors, here $90 \%$ of the measurement errors are below $8.4^{\circ}$ for the measured antenna response and below $5^{\circ}$ for the one obtained by in-field calibration. The performance using in-field calibration is clearly superior. This proofs the effectiveness of the proposed in-field calibration algorithm from Section III.

\section{CONCLUSION}

In this paper, we present an in-field calibration algorithm based on wavefield modeling and manifold separation, which is suitable for arbitrary multiport antennas. Starting with an apriori known antenna response with Gaussian model errors, the algorithm uses a MAP approach to learn an improved antenna response based on received signals and a noisy DoA reference. Effectiveness of the algorithm is demonstrated by measurements. For that, three static transmitters and a multi-mode

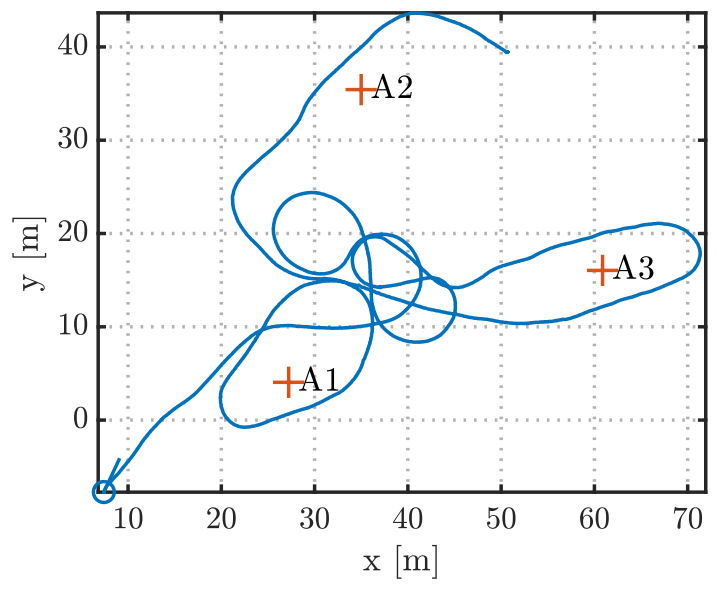

Fig. 3. Map of the evaluation scenario. A1, A2 and A3 are static anchors, the rover started driving in the lower left corner.

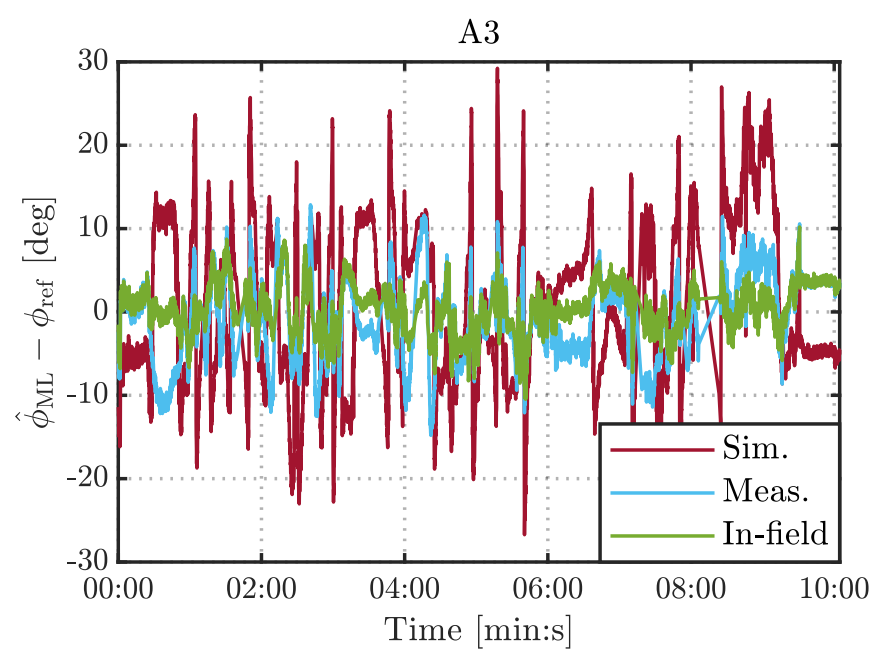

Fig. 4. DoA estimation error with respect to anchor A3 using the antenna response from EMF simulation (Sim.), measured in a near-field chamber (Meas.) and obtained by the in-field calibration algorithm from Section III (In-field).

antenna assembly are mounted on a turntable and placed in the field. Using the in-field calibration algorithm, an improved antenna response is obtained. For evaluation, a second set of measurement data is obtained, for which the antenna assembly is mounted on a rover. Using the antenna response obtained by in-field calibration, the DoA estimation performance improves by the order of several degrees compared to the antenna response obtained in a measurement chamber. With in-field calibration, $90 \%$ of the measurement errors are below $4.9^{\circ}$ to $7.9^{\circ}$ for different transmitters.

\section{ACKNOWLEDGMENT}

This work was supported by the Helmholtz Association Project "Autonomous Robotic Networks to Help Modern Societies (ARCHES)" under Grant ZT-0033 and the German Research Foundation (DFG) under Contracts FI 2176/1-2 and HO 2226/17-2. 


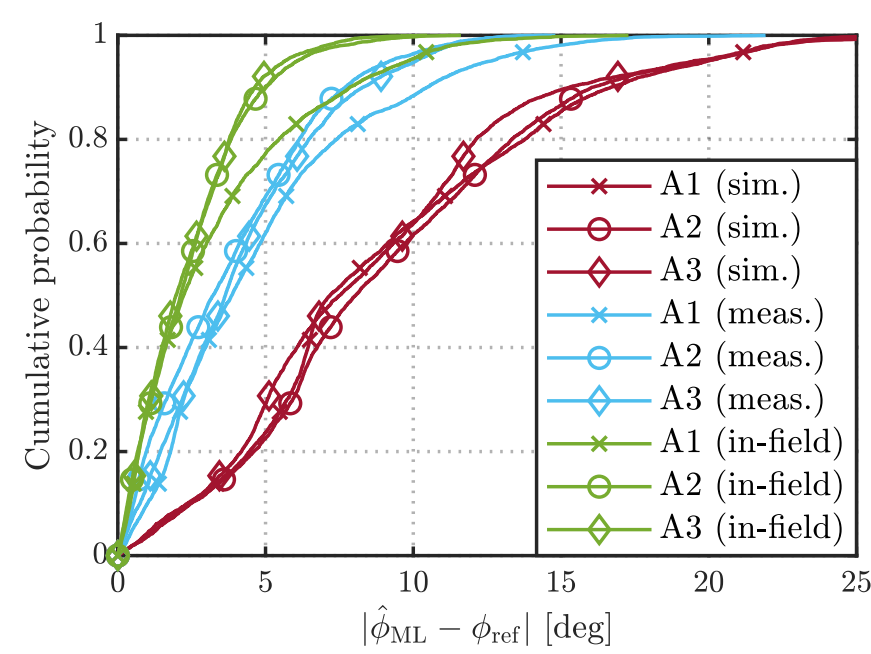

Fig. 5. Empirical cumulative probability of the DoA estimation error for the three anchors A1, A2, A3 using the antenna response from EMF simulation (sim.), measured in a near-field chamber (meas.) and obtained by the in-field calibration algorithm from Section III (in-field).

\section{REFERENCES}

[1] J. A. del Peral-Rosado, R. Raulefs, J. A. López-Salcedo, and G. SecoGranados, "Survey of cellular mobile radio localization methods: From $1 \mathrm{G}$ to 5G," IEEE Communications Surveys \& Tutorials, vol. 20, no. 2, pp. 1124-1148, Secondquarter 2018.

[2] S. Kuutti, S. Fallah, K. Katsaros, M. Dianati, F. Mccullough, and A. Mouzakitis, "A survey of the state-of-the-art localization techniques and their potentials for autonomous vehicle applications," IEEE Internet of Things Journal, vol. 5, no. 2, pp. 829-846, Apr. 2018.

[3] S. Zhang, R. Pöhlmann, T. Wiedemann, A. Dammann, H. Wymeersch, and P. A. Hoeher, "Self-aware swarm navigation in autonomous exploration missions," Proceedings of the IEEE, vol. 108, no. 7, pp. 1168 1195, Jul. 2020.

[4] M. J. Schuster, M. G. Müller, S. G. Brunner, H. Lehner, P. Lehner, R. Sakagami, A. Dömel, L. Meyer, B. Vodermayer, R. Giubilato, M. Vayugundla, J. Reill, F. Steidle, I. von Bargen, K. Bussmann, R. Belder, P. Lutz, W. Stürzl, M. Smisek, M. Maier, S. Stoneman, A. Fonseca Prince, B. Rebele, M. Durner, E. Staudinger, S. Zhang, R. Pöhlmann, E. Bischoff, C. Braun, S. Schröder, E. Dietz, S. Frohmann, A. Börner, H.-W. Hübers, B. Foing, R. Triebel, A. Albu-Schäffer, A. Wedler, J. Roberts, and G. Ishigami, "The ARCHES space-analogue demonstration mission: Towards heterogeneous teams of autonomous robots for collaborative scientific sampling in planetary exploration," IEEE Robotics and Automation Letters, pp. 1-1, 2020.

[5] R. J. Mailloux, Phased Array Antenna Handbook, 2nd ed., ser. Artech House Antennas and Propagation Library. Boston: Artech House, 2005.

[6] R. Pöhlmann, S. A. Almasri, S. Zhang, T. Jost, A. Dammann, and P. A. Hoeher, "On the potential of multi-mode antennas for direction-of-arrival estimation," IEEE Transactions on Antennas and Propagation, vol. 67, no. 5, pp. 3374-3386, May 2019.

[7] A. J. Weiss and B. Friedlander, "Array shape calibration using sources in unknown locations-a maximum likelihood approach," IEEE Transactions on Acoustics, Speech, and Signal Processing, vol. 37, no. 12, pp. 1958 1966, Dec. 1989.

[8] M. Viberg and A. L. Swindlehurst, "A Bayesian approach to autocalibration for parametric array signal processing," IEEE Transactions on Signal Processing, vol. 42, no. 12, pp. 3495-3507, Dec. 1994.

[9] M. Jansson, A. L. Swindlehurst, and B. Ottersten, "Weighted subspace fitting for general array error models," IEEE Transactions on Signal Processing, vol. 46, no. 9, pp. 2484-2498, Sep. 1998.

[10] D. Astely, A. L. Swindlehurst, and B. Ottersten, "Spatial signature estimation for uniform linear arrays with unknown receiver gains and phases," IEEE Transactions on Signal Processing, vol. 47, no. 8, pp. 2128-2138, Aug. 1999.
[11] A. J. Weiss and B. Friedlander, "Almost blind" steering vector estimation using second-order moments," IEEE Transactions on Signal Processing, vol. 44, no. 4, pp. 1024-1027, Apr. 1996.

[12] R. Pöhlmann, S. Zhang, and A. Dammann, "In-field calibration of antennas or antenna arrays using wavefield modeling," in Proc. 53rd Asilomar Conf. Signals, Systems, and Computers, Pacific Grove, USA, Nov. 2019.

[13] B. Elnour and D. Erricolo, "A novel colocated cross-polarized two-loop PCB antenna in the ISM 2.4-GHz band," IEEE Antennas and Wireless Propagation Letters, vol. 9, pp. 1237-1240, 2010.

[14] C. A. Balanis and P. I. Ioannides, Introduction to Smart Antennas. San Rafael: Morgan \& Claypool Publishers, 2007.

[15] M. Viberg and A. Zoubir, Array and Statistical Signal Processing, ser Academic Press Library in Signal Processing. Boston: Academic Press, 2014.

[16] M. A. Doron and E. Doron, "Wavefield modeling and array processing. I. Spatial sampling," IEEE Transactions on Signal Processing, vol. 42, no. 10, pp. 2549-2559, 1994.

[17] M. Costa, A. Richter, and V. Koivunen, "Unified array manifold decomposition based on spherical harmonics and 2-D fourier basis," IEEE Transactions on Signal Processing, vol. 58, no. 9, pp. 4634-4645, Sep. 2010.

[18] F. Belloni, A. Richter, and V. Koivunen, "DoA estimation via manifold separation for arbitrary array structures," IEEE Transactions on Signal Processing, vol. 55, no. 10, pp. 4800-4810, Oct. 2007.

[19] R. Fletcher, Practical Methods of Optimization. Chichester: Wiley, 1987.

[20] S. Caizzone, M. S. Circiu, W. Elmarissi, and C. Enneking, "All-GNSSband DRA antenna for high-precision applications," in Proc. 12th European Conf. Antennas and Propagation (EuCAP), Apr. 2018, pp. 543-547.

[21] E. Staudinger, D. Shutin, C. Manss, A. Viseras, and S. Zhang, "Swarm technologies for future space exploration missions," in Proc. I-SAIRAS '18: Int. Symp. Artificial Intelligence, Robotics and Automation in Space, Madrid, Spanien, Jun. 2018.

[22] R. Pöhlmann, G. Pedregosa, S. Caizzone, E. Staudinger, and P. A Hoeher, "Multi-mode antenna enabled direction-of-arrival estimation for swarm navigation," in Proc. 16th Workshop Positioning, Navigation and Communications (WPNC), Oct. 2019, pp. 1-6.

[23] ANavS GmbH, "ANavS Multi-Sensor RTK Module," https://www.anavs.de. 